\title{
The unboundedness of Hausdorff operators on Quasi-Banach spaces
}

Weichao Guo ${ }^{1}$, Jianmiao Ruan ${ }^{2}$, and Guoping Zhao ${ }^{3}$

${ }^{1}$ Jimei University

${ }^{2}$ Zhejiang International Studies University

${ }^{3}$ Xiamen University of Technology

July 4, 2020

\begin{abstract}
In this note, we show that the Hausdorff operator $\$ \mathrm{H}_{-}\{\backslash \mathrm{Phi}\} \$$ is unbounded on a large family of Quasi-Banach spaces, unless $\$ \mathrm{H}_{-}\{\backslash \mathrm{Phi}\} \$$ is a zero operator.
\end{abstract}

\section{Hosted file}

unbounded_V2.pdf available at https://authorea.com/users/339757/articles/465907-theunboundedness-of-hausdorff-operators-on-quasi-banach-spaces 